\title{
Calcium channel blockers cannot prevent pure vasospastic myocardial infarction
}

\author{
P. Depelchin and S. Degre
}

Department of Medical Cardiology, Hôpital Académique Erasme, Universite Libre de Bruxelles, 808 route de Lennik, 1070 Bruxelles, Belgium.

\begin{abstract}
Summary: Coronary artery spasm is a recognized cause of myocardial infarction. This report describes a case of myocardial infarction attributed to pure coronary spasm which was halted by a double perfusion with streptokinase and nitroglycerin. Further coronary artery spasm leading to a myocardial infarction could not be avoided several weeks later, although the patient was left on calcium channel blocker therapy. The two attacks were not preceded by warning angina pectoris, contrary to accepted belief. The best objective of end-point drug therapy and its assessment in vasospastic angina are discussed.
\end{abstract}

\section{Introduction}

Coronary artery spasm alone or superimposed on an atheromatous lesion is now recognized as a cause of myocardial infarction. ${ }^{1}$ Our therapeutic approach has therefore been directed not only towards treating factors which increase myocardial oxygen demand but also to modulating smooth-muscle contraction responsible for the coronary artery spasm. Calcium channel blockers and nitroglycerin compounds are potent smooth-muscle relaxing drugs and are effective in suppressing spasm-induced angina. However, nitroglycerin, even intracoronary administered after induced coronary spasms, has sometimes been unable to reverse the spasm. ${ }^{2}$ We present a patient free from arteriosclerotic coronary artery disease who developed pure vasospastic myocardial infarction in spite of calcium channel blocking therapy.

A 63 year old woman was admitted to the coronary care unit (CCU) with constrictive precordial pain. Serial electrocardiograms (ECG) demonstrated hallmarks of an evolving inferior myocardial infarction: ST segment elevation with upward convexity and inverted T wave in lead II, III, and aVF, reciprocal ST segment depression in anterior leads. Those modifications were refractory to sublingual dinitrate isosorbide administration (Figure 1). The patient was therefore put on a peripheral streptokinase thrombolysis

Correspondence: P. Depelchin, M.D., rue Vilain XIIII, \# 32 1050 Bruxelles, Belgium.

Accepted: 4 August 1986

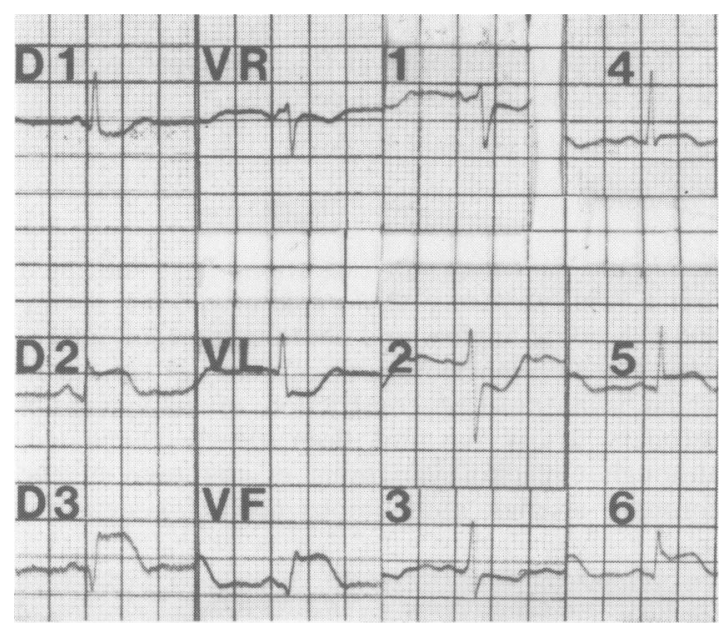

Figure 1 Electrocardiogram at entry. The tracing shows marked ST segment elevation in leads II, III, aVF and V5, V6.

programme routinely used in our hospital for impending inferior wall myocardial infarction. A concomitant gradually increased intravenous nitroglycerin drip was also begun because a coronary vasospasm could not definitely be excluded. Precordial pain was only relieved 6 hours after the beginning of thrombolysis and 8 hours after the beginning of the pain. At this moment, a non-sustained ventricular tachycardia, probably due to reperfusion, justified institution of an antiarrhythmic treatment; anticoagulation therapy with heparin was also instituted. Serial cardiac enzyme

(C) The Fellowship of Postgraduate Medicine, 1987 
levels showed only mild elevation (twice the normal range). Coronary arteriography and contrast ventriculography carried out 2 days later revealed a coronary artery tree free from atherosclerotic lesions and an almost normally contracting left ventricle. Right coronary artery spasm was suspected, but an ergonovine test was not carried out because of its hazards. The patient was put on nifedipine $10 \mathrm{mg}$ three times/day. The one week hospital course was uneventful: no relapsing precordial discomfort nor abnormality on a 3-day ECG monitoring survey in the CCU were described. A 24-hour dynamic electrocardiogram recording (Holter) also failed to show signs of a persistent vasospasm. The patient was therefore allowed to return home with the same calcium blocking agent regimen (nifedipine $10 \mathrm{mg}$ three times/day).

One month later following discharge the patient was readmitted because of a sudden constrictive precordial pain arising at rest and which, this time, led to an inferior wall infarction. No warning anginal pain was described and the patient regularly took her tablets. A subsequent ergonovine test taken under cineangiographic control demonstrated right coronary artery spasm and an inferior akinesia (Figure 2). At the time of discharge the patient was free of congestive heart disease and residual angina pectoris.

\section{Discussion}

In recent years many investigations have indicated that coronary spasms play a role in variant, resting and exertional angina, and acute myocardial infarction. The pathophysiology of coronary artery spasm which is a transient constriction of an epicardial coronary artery has not been clearly elucidated, although circadian variations of the tone of the coronary arteries, autonomous nervous system im- balance and regulation of coronary artery tone by various vasoactive substances are clearly demonstrated. ${ }^{3}$ Whatever the real mechanism(s) of spasms, the final common pathway involves an increase in intracellular calcium. Calcium antagonists block the $\mathrm{Ca}^{2+}$-channels and suppress the entry of $\mathrm{Ca}^{2+}$ into the cell. They diminish the membrane permeability of coronary artery smooth-muscle cells involved in the spasm and have progressively gained wide-spread acceptance in the treatment of coronary heart disease. Spasm may be sufficiently severe to resist sublingual or even intravenous nitroglycerin administration, and be only reversed by intracoronary nitroglycerin administration. ${ }^{2}$ Experimental studies and some clinical observations suggest that the coronary vasospasm can induce endothelial cell damage and thrombus formation which together lead to myocardial infarction. ${ }^{4}$ The right coronary spasm of our patient was severe enough to require 6 hours peripheral thrombolysis and intravenous nitroglycerin therapy. Only a limited myocardial infarction could be demonstrated by electrocardiogram (diminished $\mathbf{R}$ wave without a true $\mathbf{Q}$ necrosis wave), plasma enzyme screening and contrast ventriculography. The patient subsequently developed myocardial infarction in the assumed spastic coronary area in spite of a prophylactic dose of nifedipine.

Because the coronary arteries were free from atherosclerotic lesions and right coronary spasm was demonstrated on a post-infarction angiogram, we suggest that a vasospastic process produced the infarction. Although the case report represents an anecdotal incident and modest doses of nifedipine were used, we believe it calls for some more general considerations.

Despite continuous intravenous nitroglycerin infusion at a rate of $300 \mu \mathrm{g} / \mathrm{min}$ accompanying a streptokinase infusion, reperfusion was only achieved 6 hours later. Late onset reperfusion after intravenous thrombolysis is quite uncommon in our experience
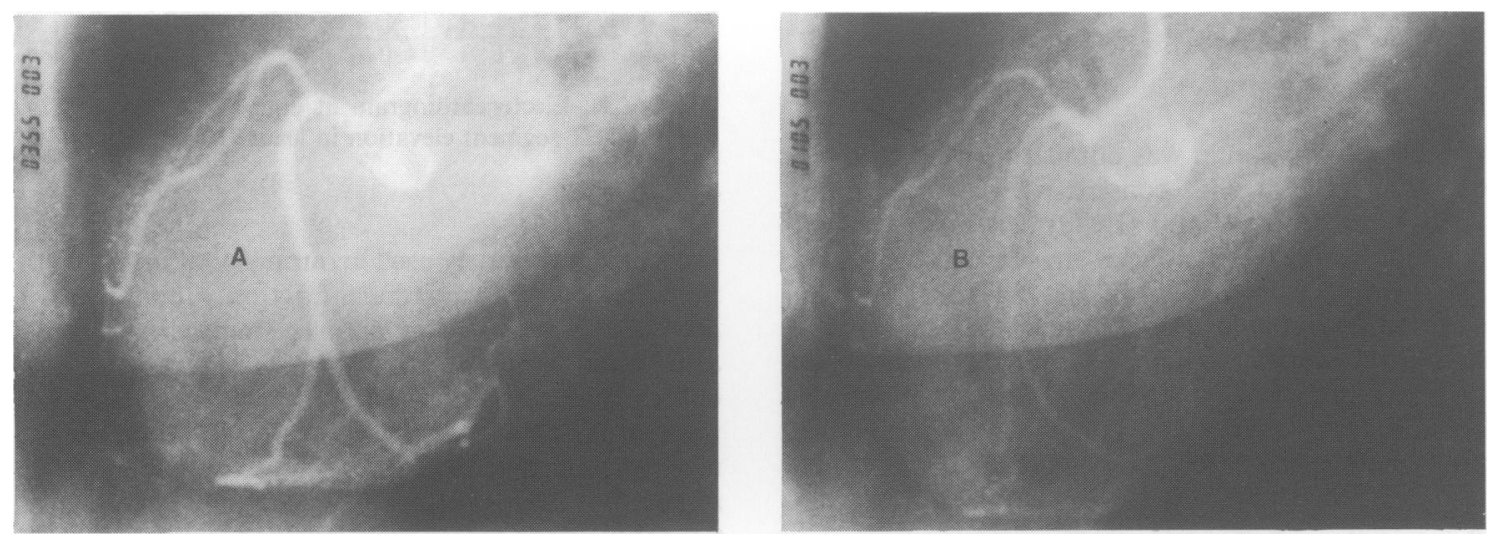

Figure 2 Coronary arteriography carried out after occurrence of the myocardial infarct shows patent left and right coronary arteries. After ergonovine test the right coronary artery (A) discloses diffuse vasospasm (B). 
and in the literature. ${ }^{5}$ This author showed that the average time from the beginning of the infusion of streptokinase to reperfusion is about 54 minutes. This observation adds a fortiori support to the statement of Buxton et $a l^{2}$ that vasospasms may be sometimes sufficiently potent to resist intravenous infusion and require intracoronary nitroglycerin administration to disappear.

Although the 3-day ECG monitoring survey in the CCU was uneventful and the patient was asymptomatic on nifedipine $10 \mathrm{mg} 3$ times/day, the occurrence of a subsequent myocardial infarction was not avoided. Therefore, suppression of attacks of pain obviously could not be the objective end-point of drug therapy. Vasospastic angina includes symptomatic attacks as well as attacks without symptoms, which in some patients may be more numerous than those with symptoms. ${ }^{4}$ Many patients with vasospastic angina will have spontaneous remission, and circadian variation of the tone of coronary arteries has been demonstrated; both facts make evaluation of drug therapy difficult. The ideal result of treatment would be total freedom from symptomatic, as well as asymptomatic, attacks of myocardial ischaemia. Because a cure is not always possible, the relief of symptoms to the point that they no longer significantly interfere with the patient's life and minimize the chance of a lifethreatening attack is a reasonable goal. There is therefore a need for a practical and sensitive test for therapeutic evaluation. Because not all attacks of myocardial ischaemia are symptomatic, it is wise to check a 24-hour ambulatory ECG recording at the beginning of the therapy and again after symptomatic attacks are controlled in order to detect asymptomatic ischaemic episodes. The Holter ECG recording is the only way to ascertain actual disease control in patients rendered asymptomatic by drug therapy. Curry et al. ${ }^{6}$ and Freeman et al. ${ }^{7}$ proposed exercise testing in selected cases as a guide to therapy, while Waters et al. ${ }^{8}$ used an ergonovine provocation test to determine if a patient was still subject to attacks of coronary artery spasm.

It has been said that patients developing myocardial infarction due to coronary artery spasm in the absence of fixed severe obstruction suffer typical attacks of angina at rest associated with transient ST segment elevation in the leads which reflect the area of infarction. ${ }^{9}$ The same author also stated that in patients with normal coronary arteriograms who develop a myocardial infarction as an isolated clinical event, coronary artery spasm was never demonstrated. Different mechanisms have been proposed to explain this kind of myocardial infarction, such as coronary embolus or thrombosis with recanalization. ${ }^{10,11}$ Our case undoubtedly suggests that coronary spasm occurring with a normal coronary arteriogram could result in myocardial infarction without warning angina. The satisfactory technical quality of angiograms and use of multi-incidental views leaves no doubt about the accuracy of the method for assessing right coronary artery patency. ${ }^{12}$

What is an optimal drug schedule? What is good for most is not necessarily the best for everyone and in the setting of vasospasm it must be remembered that even the most effective vasodilators will do no good if they are not present in effective concentrations at times when the patient is susceptible to attacks. Muller \& Gunther ${ }^{13}$ have demonstrated the ineffectiveness of low doses of $\mathrm{Ca}^{2+}$-blockers approximately 4 hours after administration. A 3- or 4-times-a-day supportive schedule ordinarily used with calcium channel blockers may not be appropriate for every patient. The omission of this pharmacological feature may explain nifedipine therapy failure in our patient since vasoconstricting adverse environmental influences favouring spasm (smoking, aspirin and other cyclo-oxygenase inhibitors, sudden withdrawal from organic nitrates, ergot alkaloids, beta-adrenergic antagonists, parasympathicomimetics and alpha-adrenergics) have been ruled out. The possibility should however be considered that, besides intracellular $\mathrm{Ca}^{2+}$ level, other mechanisms might regulate coronary artery spasm. In particular, maintenance and strengthening of the spasm by endothelial cell damage, platelet aggregates and thromboxane production are suspected. ${ }^{14}$ Such lesions, less easily reversed under spasmolytic therapy, could also explain some failure of the intravenous nitroglycerin infusion.

\section{Conclusions}

Vasospasms may sometimes be sufficiently potent to require intracoronary nitroglycerin administration to disappear. Evaluation of vasospastic angina drug therapy is difficult but suppression of attacks of pain could obviously not be the objective end-point of therapy because patients suffer an unknown number of asymptomatic attacks of myocardial ischaemia episodes. A 4-times-a-day $\mathrm{Ca}^{2+}$-channel blocker supportive schedule may not be appropriate for every patient because decreased drug concentrations 4 hours after oral administration may leave patients susceptible to attacks. Holter 24-hour ECG recording seems the most appropriate technique to ascertain actual control of the disease because prevention of myocardial ischaemia rather than prevention of recurrent precordial pain attacks should be the goal of therapy.

The case suggests also that myocardial infarction induced by coronary vasospasm may occur in a coronary artery tree totally free of fixed atheromatous obstruction, and, secondly, that this kind of pure vasospastic myocardial infarction can occur without any warning angina. 


\section{References}

1. Vincent, G.M., Anderson, J.L. \& Marshall, H.W. Coronary spasm producing coronary thrombosis and myocardial infarction. N Engl J Med 1983, 309: 220-223.

2. Buxton, A., Goldberg, S., Hirshfeld, J.W. et al. Refractory ergonovine-induced coronary vasospasm: importance of intracoronary nitroglycerin. Am J Cardiol 1980, 46(2): 329-334.

3. Yasue, H. Coronary artery spasm and calcium ions. In Opie, L.H. (ed) Calcium Antagonists and Cardiovascular Disease. Raven Press, New York, 1984, pp. 117-128.

4. Maseri, A., Servi, S., Nes, M. et al. 'Variant' angina: one aspect of a continuous spectrum of vasospastic myocardial ischemia. Am J Cardiol 1978, 42(6): 1019-1035.

5. Blunda, M., Wolf, N.M., Singh, S. et al. Intravenous $v s$ intracoronary streptokinase to re-open occluded coronary arteries - preliminary results. Circulation 1982, 66: (suppl II), 184 (Abst).

6. Curry, R.C., Pepine, C.J. \& Conti, R. Ambulatory monitoring to evaluate therapy results in variant angina patients. Circulation 1979, 60: (suppl II): 190 (Abst).

7. Freeman, B., Dunn, R.F., Richmond, D.R., \& Kelly, D.T. Coronary artery spasm during exercise: treatment with verapamil. Circulation 1981, 64: 68-75.

8. Waters, D.D., Szlachcic, J., Theroux, P., Dauwe, F. \& Mizgala, H.F. Ergonovine testing to detect spontaneous remissions of variant angina during long-term treatment with calcium antagonist drugs. Am J Cardiol 1981, 47: 179-184.

9. Heupler, F.A., Proudfit, W.L., Razavi, M., Shirey, E.K., Greenstreet, R. \& Sheldon, W.C. Ergonovine maleate provocative test for coronary artery spasm. Am J Cardiol 1978, 41: 631-640.

10. O'Reilly, R.J. \& Spellberg, W.L. Rapid resolution of coronary arterial emboli: myocardial infarction and subsequent normal coronary arteriograms. Am J Cardiol 1974, 81: 348-000.

11. Rosenblatt, A. \& Selzer, A. The nature and clinical features of myocardial infarction with normal coronary arteriograms. Circulation 1977, 55: 578-580.

12. Kemp, H.G. Coronary arteriography: indications techniques and morbidity. In Brest, A.N. (ed) Invasive Cardiology, Cardiovascular clinics. F.A. Davis, Philadelphia, 1984, pp. 1-15.

13. Muller, J.E. \& Gunther, S.J. Nifedipine therapy for Prinzmetal's angina. Circulation 1978, 57: 137-139.

14. Hillis, L.D., Hirsh, P.D., Campbell, W.B. \& Firth, G.F. Interaction of the arterial wall, plaque, and platelets in myocardial infarction. In Brest, A.N. (ed) Coronary Spasm and Thrombosis, Cardiovascular clinics. F.A. Davis, Philadelphia, 1983, pp. 31-44. 